\title{
Xampling: Analog Data Compression
}

\author{
Moshe Mishali and Yonina C. Eldar \\ Department of Electrical Engineering \\ Technion - Israel Institute of Technology, Haifa 32000, Israel \\ moshiko@tx.technion.ac.il,yonina@ee.technion.ac.il
}

\begin{abstract}
We introduce Xampling, a design methodology for analog compressed sensing in which we sample analog bandlimited signals at rates far lower than Nyquist, without loss of information. This allows compression together with the sampling stage. The main principles underlying this framework are the ability to capture a broad signal model, low sampling rate, efficient analog and digital implementation and lowrate baseband processing. In order to break through the Nyquist barrier so as to compress the signals in the sampling process, one has to combine classic methods from sampling theory together with recent developments in compressed sensing. We show that previous attempts at sub-Nyquist sampling suffer from analog implementation issues, large computational loads, and have no baseband processing capabilities. We then introduce the modulated wideband converter which can satisfy all the Xampling desiderata. We also demonstrate a board implementation of our converter which exhibits sub-Nyquist sampling in practice.
\end{abstract}

\section{Introduction}

Signal processing methods have changed substantially over the last several decades. The number of operations that are shifted from analog to digital is constantly increasing, leaving amplifications and fine tunings to the traditional front-end. Analog to digital convertors (ADC) serve as the gate to the digital domain. An ADC device outputs a stream of numbers, representing the signal amplitudes at the sampling points. These pointwise samples of the input are typically acquired at a constant rate, which traditionally is equal (or larger than) twice the highest frequency component of the signal, termed the Nyquist rate.

Digital signal processing (DSP) is the crowning glory of the chain of blocks in Fig. 1. The prime goal of the ADC is isolating the delicate interaction with the continuous world, so that sophisticated algorithms can be developed in a flexible software environment. Digital filtering, channel equalization, system identification, and a rich variety of software algorithms - all lie under the DSP block of Fig. 1. Data compression exploits redundancy in the input signal in order to reduce the storage 


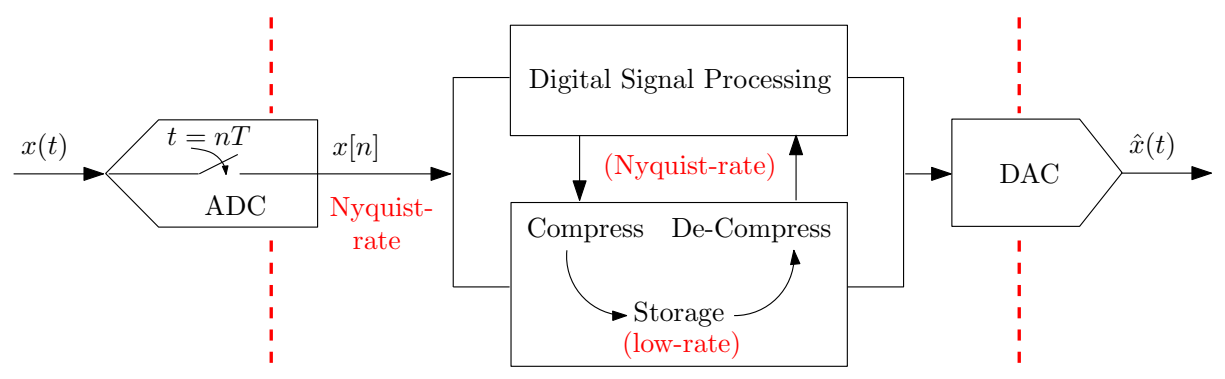

Fig. 1: Conventional blocks in a DSP system.

volume of the signal. The vast majority of DSP algorithms require the uncompressed version of the signal, namely at the Nyquist rate.

In the chain of sampling, processing and reconstruction, the conversion to digital has become a serious bottleneck. While technology advances enable mass processing of huge data streams, the acquisition capabilities do not scale sufficiently fast [1]. For some applications, the Nyquist rate already exceeds the possible rates achievable with existing devices $[2,3]$. Another rate limitation stems from the digital processor, whose clock frequency dictates the maximal input rate.

In this paper, we present Xampling - a methodology for sub-Nyquist signal acquisition, which aims at breaking through the rate bottleneck in the ADC and DSP blocks of Fig. 1. Xampling is a combination of compression and sampling: it results in samples representing the signal at a compressed rate, namely, far below Nyquist. This allows compression to be integrated into the sampling stage. Our framework consists of four criteria: broad analog signal model, low sampling rate, efficient analog and digital implementation and low-rate DSP capability. Fig. 2 visualizes the general structure of a Xampling-compliant system. Two important ingredients are: (I) the $\mathrm{X}-\mathrm{ADC}$ converts the input at a sub-Nyquist rate, and in contrast to Fig. 1 does not take pointwise samples of the input (which entailed analog bandwidth issues); (II) the DSP device operates at the low rate and does not necessitate interpolation to the Nyquist grid. Storage can also be implemented directly, without the need for preceding digital compression.

Although this paradigm is applicable to a broad class of input signals (see for example [4] which demonstrates an application to efficient time-delay estimation from sub-Nyquist samples, or [5]), for concreteness, here we consider the case of multiple narrowband transmissions located anywhere below a maximal frequency $f_{\max }$. A common approach to rate reduction in this setting is demodulation, a process which isolates an individual narrowband transmission of interest by shifting the contents from the high frequencies to the origin. Demodulation requires knowing the exact carrier frequency. Nonuniform pointwise sampling as an alternative to demodulation when the carrier frequencies are known was considered in $[6,7]$, and recently utilized even for the case of unknown transmission locations [8]. Unfortunately, pointwise strategies can be almost as difficult to implement as Nyquist samplers due to analog bandwidth limitations of existing ADC devices: in the sampling process the ADC must be exposed to the entire wideband input; see [9] for a detailed discussion. Re- 


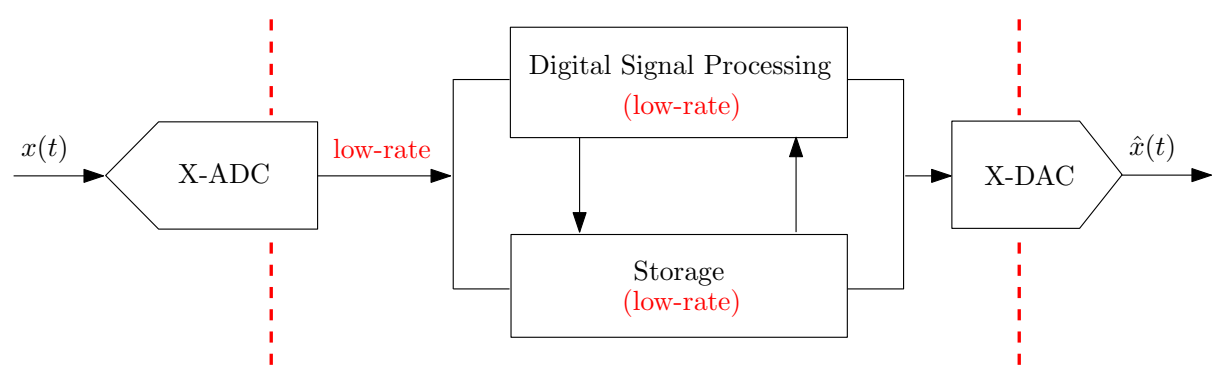

Fig. 2: The configuration of a Xampling signal processing system.

cently, the random demodulator (RD) [10] was suggested for sub-Nyquist systems which is based on exploiting ideas from the field of compressed sensing (CS) [11,12]. Mainstream CS works study the problem of recovering a sparse vector from an underdetermined linear system. The RD extends the CS ideas to sampling of signals comprised of a discrete set of harmonics. Unfortunately, the RD relies on a sensitive signal model when dealing with analog signals, the time-domain approach boils down to difficult implementation requirements and the computational complexity is severe, so that it does not satisfy the Xampling criteria.

To satisfy the Xampling properties, we introduce the modulated wideband converter (MWC) of [9]. As we show, the MWC naturally fits analog models, the implementation [13] is based on the standard frequency-domain viewpoint and the required computational complexity is by orders of magnitude smaller compared with the RD. Furthermore, baseband DSP capabilities can easily be incorporated. We also demonstrate a hardware implementation of the MWC, which exhibits sub-Nyquist sampling in practice.

\section{Xampling}

The main theme of Xampling is that a sub-Nyquist system should satisfy all four principles defined below in order to break through the Nyquist barrier.

Signal model (X1). The system should be able to handle a broad set of inputs with different spectral contents without altering either the hardware or the digital software. For communication signals, the goal should be treating analog signals with arbitrary carrier positions $f_{i}$ until $f_{\max }$.

Sampling rate (X2). The conversion rate should be as low as possible. This criterion is violated if the $\mathrm{X}-\mathrm{ADC}$ has practical limitations which prevents sampling at the minimal theoretical rate.

Efficient implementation (X3). This criterion refers to the ability to realize the X-ADC with a reasonable number of existing analog devices. It also refers to the computational burden in the digital domain, which should be as light as possible.

Baseband processing (X4). A sub-Nyquist system should enable processing of the information contents at a low rate. Baseband processing at the low rate is perhaps the most practical property of sub-Nyquist systems to consider, since perfect reconstruction of the original high-rate analog $x(t)$ is often less useful. 
Exploiting sparsity for rate reduction has been explored in the context of CS, which treats underdetermined sparse recovery. The signal model assumes a vector $\mathbf{x}$ of finite length $n$, which has only a few nonzero entries. Sensing is carried out by computing the linear projection $\mathbf{y}=\mathbf{A x}$, with $\mathbf{A}$ having far fewer rows than columns. Results from this field $[11,12]$ show that under suitable conditions, the linear sensing is stably invertible, even when the length of $\mathbf{y}$ is proportional to the number of nonzeros in $\mathbf{x}$, rather than the ambient dimension $n$. However, it is not straightforward to generalize the discrete CS formulation to analog signals. The difficulty can be noticed immediately in the signal model. Sparsity is defined in CS by counting the number of nonzeros in $\mathbf{x}$, while analog sparsity of $x(t)$ involves an uncountable number of zeros and nonzeros. Naïve extensions of CS-type algorithms for sparse recovery to infinite dimensions leads to undefined or difficult problems [14]. Discretization methods result in very large scale CS systems, which impose a severe burden on the digital processing units.

The CS paradigm aims at avoiding high-rate redundant sampling. The discrete CS framework $[11,12]$ initiated a long line of highly influential works. However, it still remains puzzling from the analog sampling viewpoint; sensing by $\mathbf{y}=\mathbf{A x}$ implicity assumes that $\mathbf{x}$ is the Nyquist rate samples of some continuous signal $x(t)$ on a specific time-interval. We propose Xampling as a general framework within various solutions for analog signals can be compared according to the four rules (X1)-(X4).

The nomenclature Xampling was chosen to highlight the important aspects of our framework. Sub-Nyquist systems rely on the theory of sampling from a union of subspaces $[4,5,15,16]$, whereas classic results were stated for single subspace models $[17-19]$. The $\mathrm{X}$ prefix therefore distinguishes analog sub-Nyquist systems from classic results in the sampling literature, though Xampling still hints that our framework is only a sub-field of generalized sampling theory [19]. The naming has a symbolic interpretation as well. The $\mathrm{X}$ letter is widely used to denote compression, e.g. the DivX format. Here, the compression is carried out during the conversion from analog to digital, conceptually using analog means rather than by software algorithms. Finally, as we observe in the sequel, breaking-through the Nyquist barrier necessitates balancing between CS and sampling by combining traditional concepts from sampling theory together with recent CS developments. Xampling is literally pronounced as CS-Sampling (phonetically /k'sæmplıy/), so as to symbolize the necessity of this synergy in practice.

\section{The Random Demodulator}

Fig. 3(a) presents the RD of [10]. The input signal $f(t)$ is mixed by a pseudorandom sign waveform which alternates at rate $W$. The mixed output is then integrated and dumped at a constant rate $R$, resulting in the sequence $y[n], 1 \leq n \leq N_{R}$. The design parameters are the rates $W, R$ and the number of samples $N_{R}$.

The authors [10] analyze the RD through the system of Fig. 3(b), which requires certain assumptions to hold. It can be easily verified from the figure that if $R$ divides $W$, then every measurement $y[n]$ corresponds to the sum over $W / R$ consecutive 


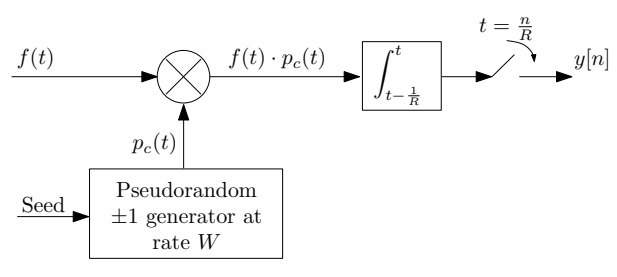

(a)

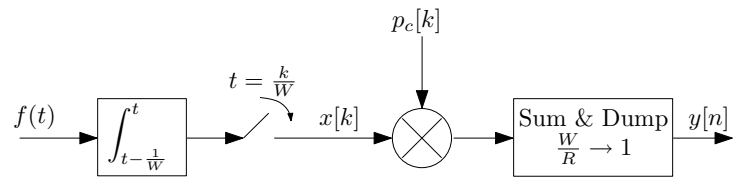

(b)

Fig. 3: Block diagram of the random demodulator (a), and the equivalent system (b) for an integer ratio $W / R$.

products $x[k] p_{c}[k]$, where $x[k]$ is obtained by integrating $f(t)$ over $1 / W$ time periods. To connect the input signal $f(t)$ to $x[k]$, a multitone model is assumed:

$$
f(t)=\sum_{\omega \in \Omega} a_{\omega} e^{j 2 \pi \omega t}
$$

where $\Omega$ is a finite set of $K$ out of an even number $Q$ of possible harmonics $\Omega \subset$ $\{0, \pm \Delta, \pm 2 \Delta, \cdots, \pm(0.5 Q-1) \Delta, 0.5 Q \Delta\}$. The sparse tones model (1) is parameterized by $K, Q$ and the spacing $\Delta$.

The equivalence between the left and right panes of Fig. 3 requires that $W=Q \Delta$. Another requirement, which will turn out to be more essential, is that

$$
Q=N_{R} \frac{W}{R}=N_{R} \frac{Q \Delta}{R} \rightarrow \Delta=\frac{R}{N_{R}}
$$

Under these selections, the $N_{R}$ samples can be collected to a vector $\mathbf{y}$, which obeys $\mathbf{y}=\boldsymbol{\Phi} \mathbf{s}$. The vector $\mathbf{s}$ is a one-to-one mapping of the unknown coefficients $a_{\omega}$ from (1), thus may have $K$ nonzero entries at the most. The matrix $\boldsymbol{\Phi}$ is defined by the pseudo-random sequence $p(t)[10]$.

Due to space limitation, we investigate the RD in the light of efficient implementation (X3), and refer the reader to the technical report [20] for further details regarding the other Xampling criteria. The finite parameterization (1) of $K$ out of $Q$ possible harmonics exhibits an inherent sensitivity; the requirement (2) forces a strict match between the (unknown) tone spacing of the input to the rates $R, W$, which are presumably set in advance. Clock circuitries may vary the basis frequency with voltage, temperature, humidity, aging and other factors, which can wreck (2). As a toy-example suppose $W=1 \mathrm{kHz}, N_{R}=R=100 \mathrm{~Hz}$ and consider the signal $f(t)=3 \cos (2 \pi 120 t)+4 \cos (2 \pi 350 t), \quad t \in[0,1)$. Applying an $\ell_{1}$ minimization [11] reconstructs $\hat{f}(t)=f(t)$ exactly. However, with $0.5 \%$ inaccuracy, so that $R^{\prime}=100.5$ $\mathrm{Hz}$ and $W^{\prime}=1.005 \mathrm{kHz}$, the reconstructed $\hat{f}(t)$ of Fig. 4(a) has normalized squarederror $\|f-\hat{f}\|^{2} /\|f\|^{2}=1.7$. The frequency contents are compared in Fig. 4(b).

In practice, signals such as narrowband transmissions contain infinitely many harmonics. In order to approximate analog multiband signals using the model $(1), K$ must be of the order the occupied spectrum bandwidth, and $W=Q$ shall be the Nyquist rate $f_{\mathrm{NYQ}}=2 f_{\max }$, both in $\mathrm{Hz}$ units due to the tone spacing $\Delta=1 \mathrm{~Hz}$. Higher spacing $\Delta$ deteriorates the representation of the analog input. Consider a 


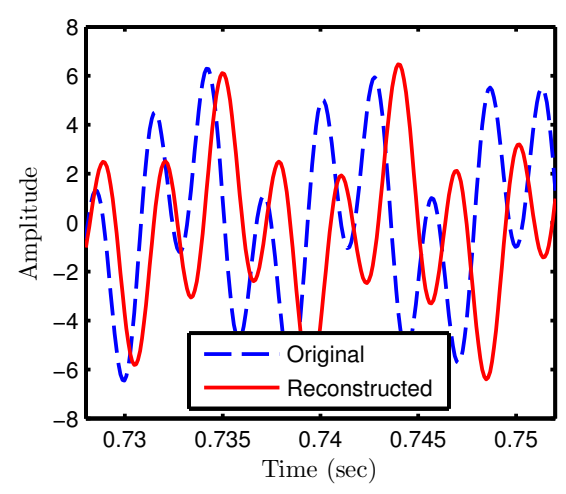

(a)

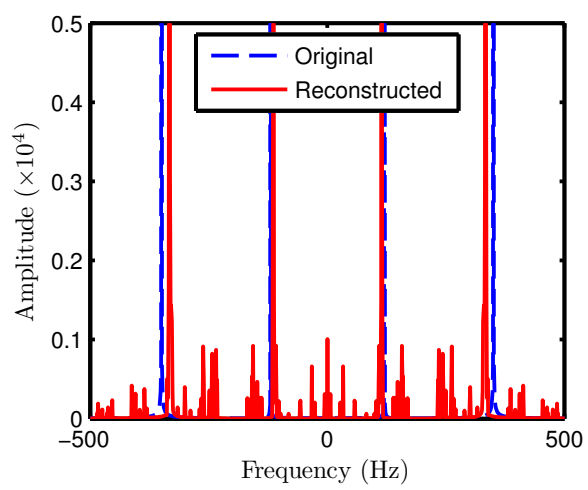

(b)

Fig. 4: Recovery of a multitone signal from RD samples under design imperfections. The original and reconstructed signal are plotted in (a) on a short time interval. The frequency transforms (b) reveal many spurious tones due to the clock shift.

wideband scenario with $N=6$ bands of width $B=50 \mathrm{MHz}$ and $f_{\mathrm{NYQ}}=10 \mathrm{GHz}$, which boils down to $K=N B=300 \cdot 10^{6}$ tones. In this setting $W=10^{10}$ and $\boldsymbol{\Phi}$ has about $R=N_{R}=2.6 \cdot 10^{9}$ rows, resulting in a huge-scale sparse recovery system. Solving a CS system with huge-scale dimensions, imposes severe computational loads. The hardware synchronization and the severe computational loads, which are associated with the huge-scale dimensions of $\boldsymbol{\Phi}$, stand against the Xampling criterion (X3).

\section{The Modulated Wideband Converter}

The MWC system overcomes the previous limitations by sticking to the traditional frequency-domain analysis, and in the same time employing CS algorithms where beneficial. This balanced combination of CS and sampling leads to an X-ADC system which satisfies (X1)-(X4).

The MWC consists of an analog front-end with $m$ channels. In the $i$ th channel, the input signal $x(t)$ is multiplied by a periodic waveform $p_{i}(t)$, lowpass filtered, and then sampled at rate $1 / T$. In this paper, we study a simplified version of the converter, as depicted in Fig. 5, in which the sampling interval $T$ equals the period of the waveforms $p_{i}(t)$. This basic configuration has three parameters: number of channels $m$, periodic waveforms $p_{i}(t)$ and sampling rate $1 / T$. Other configurations with practical advantages are detailed in [9].

The MWC sensing relies on the following key observation. The mixing operation scrambles the spectrum of $x(t)$ such that the baseband frequencies that reside below the filter cutoff $1 / 2 T$, contain a mixture of the spectral contents from the entire Nyquist range. The periodicity of each waveform $p_{i}(t)$ ensures that the mixture has a specific nature - aliases at $1 / T$ frequency spacing. Whilst aliasing is often considered as an undesired effect, here it is deliberately utilized to shift various frequency regions to baseband, simultaneously. In the basic configuration, we choose the rate $1 / T \geq B$. 


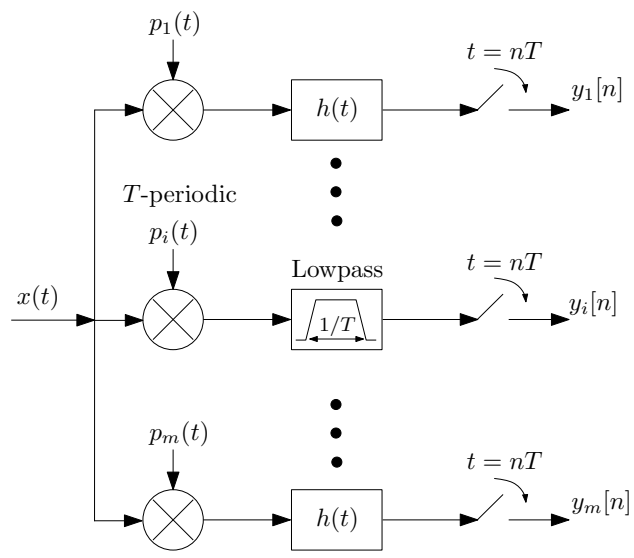

Fig. 5: Block diagram of the modulated wideband converter.

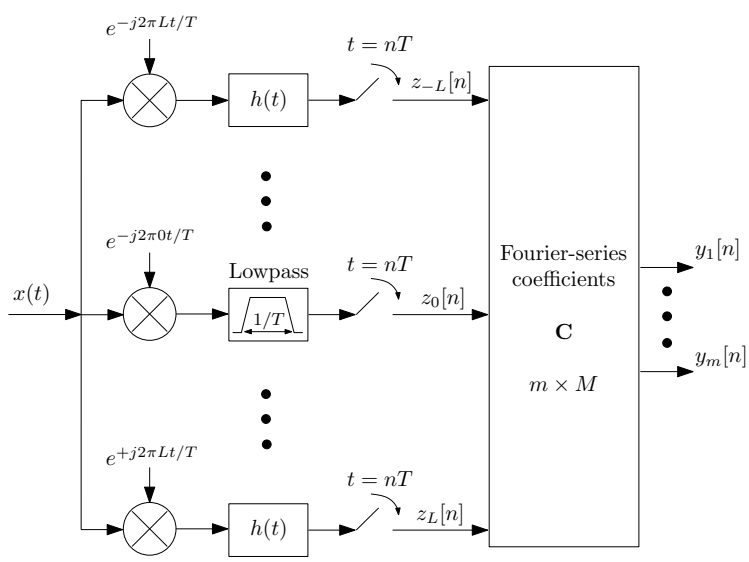

Fig. 6: The equivalent system of the modulated wideband converter.

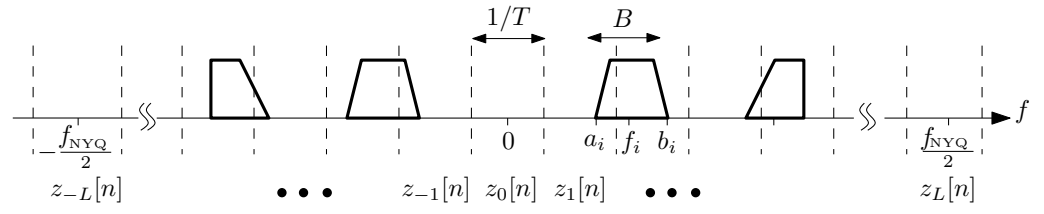

Fig. 7: The lowrate sequences $z_{l}[n]$ correspond to equal-width spectrum slices from the Fourier transform of the input multiband signal.

To understand the act of the MWC on multiband signals, we consider the equivalent system that is depicted in Fig. 6. The signal $x(t)$ enters $M=2 L+1$ channels, where $L$ is the smallest integer such that $M \geq T f_{\mathrm{NYQ}}$. Since $1 / T \approx B, M$ represents the compression ratio, that is the quotient of the Nyquist rate $f_{\mathrm{NYQ}}$ by the rate $1 / T$ of a single channel. In the $l$ th channel, $x(t)$ is frequency-shifted (hence modulated in time) by $l / T \mathrm{~Hz},-L \leq l \leq L$. Then, the baseband region $[-1 / 2 T, 1 / 2 T]$ is filtered and sampled every $T$ seconds, giving the (complex-valued) sequence $z_{l}[n]$; see the illustration in Fig. 7. Clearly, if $x(t)$ contains only a few transmissions, then most of the time sequences $z_{l}[n]$ will be identically zero. This is where CS comes into play. For each time-point, the vector $\mathbf{z}[n]=\left[z_{-L}[n], \ldots, z_{L}[n]\right]^{T}$ is compressed into the output vector $\mathbf{y}=\left[y_{1}[n], \ldots, y_{m}[n]\right]^{T}$ using the linear projection $\mathbf{y}[n]=\mathbf{C z}[n]$. The equivalence to Fig. 5 holds due to the periodicity of the waveforms $p_{i}(t)$. Since $p_{i}(t)=p_{i}(t+T)$ for all $t \in \mathbb{R}$, we have the Fourier expansion

$$
p_{i}(t)=\sum_{l=-\infty}^{\infty} c_{i l} e^{j \frac{2 \pi}{T} l t} .
$$

Choosing the matrix $\mathbf{C}$ of Fig. 6 such that its $i l$ th entry is equal to the Fourier coefficient $c_{i l}$ results in the desired equivalence

$$
\left.y_{i}[n] \stackrel{\text { Fig. }}{=}\left(x(t) p_{i}(t)\right) \star h(t)\right|_{t=n T} \stackrel{\text { Fig. } 6}{=} \sum_{l=-L}^{L} c_{i l}\left(\left.\left(x(t) e^{-j 2 \pi l t / T}\right) \star h(t)\right|_{t=n T}\right) \text {. }
$$

Conceptually, the MWC shifts the mixing matrix $\mathbf{C}=\left\{c_{i l}\right\}$ into the analog domain, 


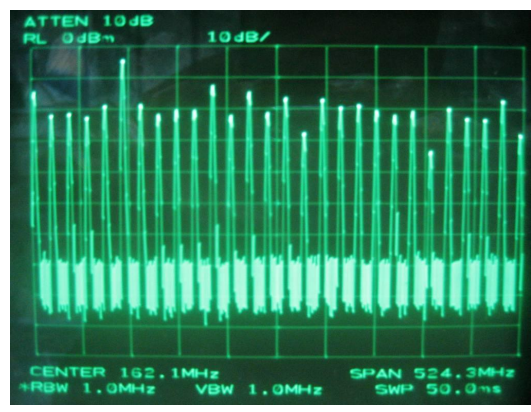

(a)

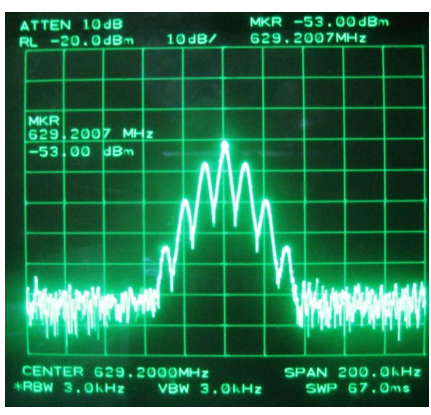

(b)

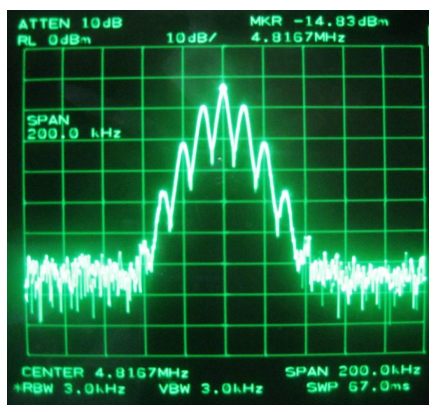

(c)

Fig. 8: The spectral contents of a single sign-alternating periodic waveform (a). A high-rate frequency-modulated input with carrier $f_{c}=629 \mathrm{MHz}$ in (b) is down-converter to $4.81 \mathrm{MHz}$ in (c). Note that the signal structure is unaltered, which enables processing of the signal directly from low rate samples [20].

such that each channel realizes a single row of $\mathbf{C}$ in analog hardware. The periodicity of $p_{i}(t)$ is the only essential requirement for the equivalence to hold.

Recovery of $x(t)$ from the sample sequences $\mathbf{y}[n]$ consists of two steps. First, recovering the frequency support at the resolution of a single slice. This step involves sparse recovery from the linear system, $\mathbf{V}=\mathbf{C U}$, where $\mathbf{V}$ is a matrix calculated from the a short-length set of consecutive samples $\mathbf{y}[n]$. The index set $S$ of the nonzero rows in the sparsest matrix solution $\mathbf{U}$ indicates the indices $l \in S$ of the slices $z_{l}[n]$ which contain signal energy. The second step is applied once the support $S$ is found, in which the pseudo-inverse $\mathbf{C}_{S}^{\dagger}$ is computed and is then used to recover $\mathbf{z}[n]$. The notation $\mathbf{C}_{S}$ means the column subset of $\mathbf{C}$ indicated by $S$. In contrast to the huge dimensions of the RD matrix $\mathbf{\Phi}$, the MWC recovery involves the matrix $\mathbf{C}$ of size $m \times M$ which is typically small, and thus the digital computations, such as the sparse recovery system $\mathbf{V}=\mathbf{C U}$ or the inversion $\mathbf{C}_{S}^{\dagger}$, are not an issue.

As with the $\mathrm{RD}$, we shall focus on the implementation aspects; further details and comparisons can be found in [20]. To begin with, note that the model underlying the MWC is an analog multiband model with $N$ bands of width $B$. As in conventional sampling, the analog signal is converted into sequences of digital samples. No finite parametrization is used.

The parameter choice of the MWC, $1 / T \geq B, M \geq T f_{\mathrm{NYQ}}$, involves only inequalities. Therefore setting $T, M$ with small safeguards allows to apply the MWC to multiband signals, even if the actual width $B$ or the frequency $f_{\max }$ are higher to some extent than what assumed in design. The approach underlying the MWC favors the classic frequency-domain viewpoint; the aliasing due to (3), the equivalence between Figs. 5,6, and the spectrum slices 7 . We have recently design a circuit which implements the MWC [13]. In our hardware realization, we took advantage of the frequency viewpoint to solve several circuit challenges, of which the design of $p_{i}(t)$ is relevant to the current scope. Our board uses $p_{i}(t)$ with sign alternations at $2 \mathrm{GHz}$ speed. Maintaining nice rectangular sign shapes at this rate is impossible. Fortunately, only the periodicity is required to ensure (3). Fig. 8(a) demonstrates the frequency response of one mixing waveform, and affirms the periodicity. As a proof 
of concept, Figs. 8(b)-(c) demonstrate how a $2 \mathrm{GHz}$ Nyquist input, is converted to a very low rate region, without knowledge of the location of the transmission.

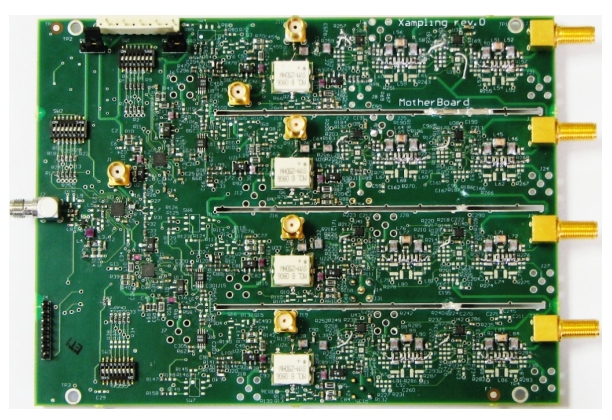

(a)

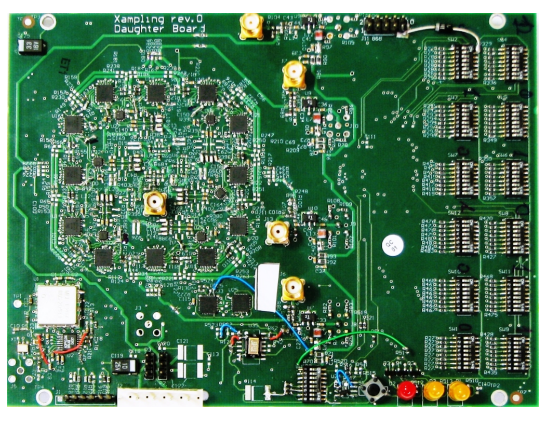

(b)

Fig. 9: The prototype board of the MWC samples $2 \mathrm{GHz}$ Nyquist-rate inputs, with only 240 $\mathrm{MHz}$ sampling rate.

From a computational aspect, the dominating cost is dictated by the size of the matrix $\mathbf{C}$. In the $\mathrm{MWC}, \mathbf{C}$ has $m$ rows, as the number of sampling channels, and $M$ columns as the number of sign alternations per period. In the board realization [13], $\mathbf{C}$ is $12 \times 200$. In fact, using several ideas from [9], we managed to achieve $m=12$ sampling sequences from only 4 analog channels, as noticed from the board photos in Fig. 9. Since the dimensions of $\mathbf{C}$ are relatively small (by orders of magnitude compared with the RD sensing matrix $\boldsymbol{\Phi}$ ), solving this CS system can be performed quite fast.

\section{References}

[1] L. Bin, T. W. Rondeau, J. H. Reed, and C. W. Bostian, "Analog-to-digital converters," IEEE Signal Process. Mag., vol. 22, no. 6, pp. 69-77, Nov. 2005.

[2] "A/D Converters," Analog Devices Corp., 2009, [Online]. Available: http://www.analog.com/en/analog-to-digital-converters/adconverters/products/index.html.

[3] "Data converters," Texas Instruments Corp., 2009, [Online]. Available: http://focus.ti.com/analog/docs/dataconvertershome.tsp.

[4] K. Gedalyahu and Y. C. Eldar, "Low rate sampling schemes for time delay estimation," arXiv.org 0905.2429, May 2009.

[5] Y. C. Eldar, "Compressed sensing of analog signals in shift-invariant spaces," IEEE Trans. Signal Process., vol. 57, no. 8, pp. 2986-2997, Aug. 2009.

[6] C. Herley and P. W. Wong, "Minimum rate sampling and reconstruction of signals with arbitrary frequency support," IEEE Trans. Inf. Theory, vol. 45, no. 5, pp. 1555-1564, Jul. 1999. 
[7] H. J. Landau, "Necessary density conditions for sampling and interpolation of certain entire functions," Acta Math., vol. 117, pp. 37-52, Feb. 1967.

[8] M. Mishali and Y. C. Eldar, "Blind multiband signal reconstruction: Compressed sensing for analog signals," IEEE Trans. Signal Process., vol. 57, no. 3, pp. 9931009, Mar. 2009.

[9] — - "From theory to practice: Sub-Nyquist sampling of sparse wideband analog signals," arXiv.org 0902.4291; to appear IEEE J. Sel. Topics Signal Process.

[10] J. A. Tropp, J. N. Laska, M. F. Duarte, J. K. Romberg, and R. Baraniuk, "Beyond nyquist: Efficient sampling of sparse bandlimited signals," arXiv.org 0902.0026, Jan. 2009.

[11] D. L. Donoho, "Compressed sensing," IEEE Trans. Inf. Theory, vol. 52, no. 4, pp. 1289-1306, April 2006.

[12] E. J. Candès, J. Romberg, and T. Tao, "Robust uncertainty principles: Exact signal reconstruction from highly incomplete frequency information," IEEE Trans. Inf. Theory, vol. 52, no. 2, pp. 489-509, Feb. 2006.

[13] M. Mishali, D. O. Eldar, Y. C., and E. Shoshan, "Xampling: Analog to digital at sub-Nyquist rates," CCIT Report no. 751, EE Dept., Technion; arXiv.org 0912.2495, Dec. 2009.

[14] M. Mishali and Y. C. Eldar, "Reduce and boost: Recovering arbitrary sets of jointly sparse vectors," IEEE Trans. Signal Process., vol. 56, no. 10, pp. 46924702, Oct. 2008.

[15] Y. C. Eldar, "Uncertainty relations for shift-invariant analog signals," arXiv.org 0809.3731; to appear IEEE Trans. Inf. Theory, Sep. 2008.

[16] Y. C. Eldar and M. Mishali, "Robust recovery of signals from a structured union of subspaces," IEEE Trans. Inf. Theory, vol. 55, no. 11, pp. 5302-5316, Nov. 2009.

[17] M. Unser, "Sampling - 50 years after Shannon," Proceedings of the IEEE, vol. 88, no. 4, pp. 569-587, Apr. 2000.

[18] P. P. Vaidyanathan, "Generalizations of the sampling theorem: Seven decades after Nyquist," IEEE Trans. Circuit Syst. I, vol. 48, no. 9, pp. 1094-1109, Sep. 2001.

[19] Y. C. Eldar and T. Michaeli, "Beyond bandlimited sampling," IEEE Signal Process. Mag., vol. 26, no. 3, pp. 48-68, May 2009.

[20] M. Mishali and Y. C. Eldar, "Xampling-Part I: Practice," CCIT Report no. 747, EE Dept., Technion; arXiv.org 0911.0519, Oct. 2009. 